\title{
Annihilation operators associated with unstable vacua in non-equilibrium thermo-field dynamics
}

\author{
K.Yoshida, T.Arimitsu \\ Graduate School of Pure and Applied Sciences, University of Tsukuba, \\ 1-1-1 Tennoudai, Ibaraki 305-8571, Japan \\ Received July 14, 2008
}

Out of thermal equilibrium state, the vacuum is unstable and evolves in time. Consequently, the annihilation operators associated with the unstable vacuum depend on time. This dissipative time-evolution of quantum systems can be systematically treated, within the canonical operator formalism referred to as non-equilibrium thermo-field dynamics. Given is an alternative route to derive the time-dependent annihilation operators within the formalism. As an example, time-dependent annihilation operators for the systems of bosonic and fermionic semi-free fields are derived.

Key words: non-equilibrium thermo-field dynamics, annihilation operator, unstable vacuum, dissipative quantum systems, time-dependent Bogoliubov transformation

PACS: $11.10 . W x, 05.30 .-d$

\section{Introduction}

One of the basic frameworks used in treating dissipative quantum systems is given by the quantum Liouville equation (the quantum master equation)

$$
\frac{\partial}{\partial t} \rho(t)=-\mathrm{i} L \rho(t)
$$

with the properties of the Liouville operator $L$,

$$
\begin{aligned}
(\mathrm{i} L \bullet)^{\dagger} & =\mathrm{i} L \bullet, \\
\operatorname{tr} L \bullet & =0,
\end{aligned}
$$

and the condition of $\rho(t)$ at the initial time $t=0: \rho^{\dagger}(0)=\rho(0)$ (see, e.g., [1] for studies of dissipative classical and quantum systems based on the Liouville equation or the Liouville-von Neumann equation). Note that $L$ is a superoperator[2], i.e., an operator acting on operators. The expectation value of an observable operator $A$ is given by

$$
\langle A\rangle_{t}=\operatorname{tr} A \rho(t)=\operatorname{tr} A \mathrm{e}^{-\mathrm{i} L t} \rho(0)=\operatorname{tr} \mathrm{e}^{\mathrm{i} L t} A \mathrm{e}^{-\mathrm{i} L t} \rho(0)=\operatorname{tr} A(t) \rho(0),
$$

where the formal solution $\rho(t)=\mathrm{e}^{-\mathrm{i} L t} \rho(0)$ of (1) and the property (3) are used. The operator $A(t)$ is defined by

$$
A(t):=\mathrm{e}^{\mathrm{i} L t} A \mathrm{e}^{-\mathrm{i} L t},
$$

and obeys the equation of motion,

$$
\frac{\mathrm{d} A(t)}{\mathrm{d} t}=\mathrm{i}[L(t), A(t)]
$$

where $L(t):=\mathrm{e}^{\mathrm{i} L t} L \mathrm{e}^{-\mathrm{i} L t}=L$.

Equations (1)-(6) form a canonical operator formalism with the superoperator $L$ being the infinitesimal time-evolution generator. Note that, in the case of non-dissipative systems, (1) reduces 
to the Liouville-von Neumann equation, i.e., the operation of $L$ reduces to $L \bullet=[H, \bullet]$ where $H$ is a Hamiltonian. However, in the presence of dissipation, the operation of $L$ is represented by a more complex entanglement of operators (see, e.g., [3]). The entanglement of operators is usually eliminated by mapping the operator equation into a partial differential equation in a certain cnumber function space (see, e.g., [4,3]).

Within the framework of Non-Equilibrium Thermo-Field Dynamics (NETFD) [5-7], the quantum Liouville equation (1) can be rewritten in the form of the Schrödinger equation (in the unit $\hbar=1)$,

$$
\frac{\mathrm{d}}{\mathrm{d} t}|0(t)\rangle=-\mathrm{i} \hat{H}|0(t)\rangle
$$

by introducing two kinds of operators, non-tilde and tilde operators ${ }^{1}$. The Schrödinger equation (7) is free from the entanglement among operators appearing in (1). The hat-Hamiltonian $\hat{H}$ consists of non-tilde and tilde operators, and $|0(t)\rangle$ is the ket-vacuum which contains the same amount of information as the density operator $\rho(t)$ (see section 2). Note that non-tilde and tilde operators (and therefore $\hat{H}$ ) are not superoperators but just ordinary operators acting on the Fock space built on the vacuum $|0(t)\rangle$. Note also that the vacuum $|0(t)\rangle$ within NETFD is unstable and dependent on time, the situation being quite different from the case in usual quantum field theory (QFT). One can introduce the annihilation operator $\gamma_{t}\left(\tilde{\gamma}_{t}\right)$ specified by $\gamma_{t}|0(t)\rangle=0\left(\tilde{\gamma}_{t}|0(t)\rangle=0\right)$ and the creation operator $\gamma^{+}\left(\tilde{\gamma}^{+}\right)$which is the canonical conjugate of $\gamma_{t}\left(\tilde{\gamma}_{t}\right)$. [The operator $\gamma^{+}\left(\tilde{\gamma}^{+}\right)$is time-independent reflecting the fact that the bra-vacuum $\langle 1|$ is time-independent in NETFD, see section 2.] One can manipulate operator algebra such as the time-ordering or the normal-ordering with respect to $\gamma_{t}, \gamma^{+}, \tilde{\gamma}_{t}$ and $\tilde{\gamma}^{+}$, in a way similar to usual QFT. Thus, NETFD provides us with a canonical operator formalism for dissipative quantum systems which preserves most of the technical properties in usual QFT.

In this paper, we show an alternative route to derive the annihilation operators $\gamma_{t}$ and $\tilde{\gamma}_{t}$. Conventionally, they were derived in relation to the time-dependent annihilation operators $\gamma(t)$ and $\tilde{\gamma}(t)$ for unstable physical particles which annihilate the initial vacuum $|0\rangle=|0(t=0)\rangle$, i.e., $\gamma(t)|0\rangle=0$ and $\tilde{\gamma}(t)|0\rangle=0 \quad[5-7,9]$ (see appendix A). The alternative route is rather similar to the Bogoliubov transformation within usual QFT, i.e., $a(\theta)=U(\theta) a U^{-1}(\theta)$ induced by a unitary operator $U(\theta)$ with a parameter $\theta$. The annihilation operators $a$ and $a(\theta)$ define, respectively, vacua $|0\rangle$ and $|0(\theta)\rangle$ through the relations $a|0\rangle=0$ and $a(\theta)|0(\theta)\rangle=0^{2}$. When a system is out of thermal equilibrium state and the vacuum $|0(t)\rangle$ is unstable, time $t$ may play the role of the parameter $\theta$. We will see that a special treatment is required when the parameter $\theta$ turns out to be time $t$ (see section 5 for details).

This paper is organized as follows. In section 2, we briefly review the formalism of NETFD. In section 3, we show the general formulation of the alternative route to derive the annihilation operators associated with the unstable vacuum. In section 4 , as an example, the annihilation operators for semi-free fields are derived through the route given in section 3 . In section 5 , we discuss the implication of the present derivation including its relation to the conventional one. The conventional derivation of the annihilation operators is given in appendix A.

\section{Preliminaries}

\subsection{Basics of NETFD}

Here we briefly review the formalism of NETFD in order to fix the notations ${ }^{3}$. Within the formalism of NETFD, any operator $A$ is accompanied by its tilde conjugate $\tilde{A}$. The operators $A$ and $\tilde{A}$ are called, respectively, non-tilde operator and tilde operator. The tilde conjugation is

\footnotetext{
${ }^{1}$ It was noticed first by Crawford [2] that, with the introduction of two kinds of operators, the Liouville-von Neumann equation for non-dissipative systems reduces to the form of the Schrödinger equation.

${ }^{2}$ For QFT in finite temperature, i.e., thermo-field dynamics (TFD)[8], the parameter $\theta$ labeling vacua is temperature.

${ }^{3}$ For more information on NETFD, see the original sources [5-7] and the review paper [10].
} 
defined by the following rules,

$$
\begin{aligned}
\left(A_{1} A_{2}\right)^{\sim} & =\tilde{A}_{1} \tilde{A}_{2}, \\
\left(c_{1} A_{1}+c_{2} A_{2}\right)^{\sim} & =c_{1}^{*} \tilde{A}_{1}+c_{2}^{*} \tilde{A}_{2}, \\
(\tilde{A})^{\sim} & =A, \\
\left(A^{\dagger}\right)^{\sim} & =\tilde{A}^{\dagger},
\end{aligned}
$$

where $A, A_{1}$ and $A_{2}$ represent operators, and $c_{1}$ and $c_{2}$ c-numbers. The tilde operators and non-tilde operators are mutually (anti-)commutative at equal time:

$$
\left[A_{1}, \tilde{A}_{2}\right]_{-\sigma}=0
$$

where $\left[A_{1}, A_{2}\right]_{-}:=\left[A_{1}, A_{2}\right]=A_{1} A_{2}-A_{2} A_{1},\left[A_{1}, A_{2}\right]_{+}:=\left\{A_{1}, A_{2}\right\}=A_{1} A_{2}+A_{2} A_{1}$, and $\sigma$ is a real parameter given by

$$
\sigma:=\left\{\begin{array}{ll}
1 & \text { (for bosonic operators) } \\
-1 & \text { (for fermionic operators) }
\end{array} .\right.
$$

The expectation value of an operator $A$ at time $t$ is given by

$$
\langle A\rangle_{t}:=\langle 1|A| 0(t)\rangle
$$

where $\langle 1|$ and $|0(t)\rangle$ are, respectively, the thermal bra-vacuum and the thermal ket-vacuum. The vacua $\langle 1|$ and $|0(t)\rangle$ are tilde invariant:

$$
\left\langle\left. 1\right|^{\sim}=\langle 1|, \quad| 0(t)\rangle^{\sim}=\mid 0(t)\right\rangle,
$$

and are normalized as

$$
\langle 1 \mid 0(t)\rangle=1
$$

Tilde and non-tilde operators are related with each other through the thermal state condition (TSC)

$$
\tau\langle 1| \tilde{A}=\langle 1| A^{\dagger},
$$

where $\tau$ is a complex parameter given by ${ }^{4}$

$$
\tau:=\left\{\begin{array}{ll}
1 & \text { (for bosonic operators) } \\
\mathrm{i} & \text { (for fermionic operators) }
\end{array}\right. \text {. }
$$

In NETFD, observable operators consist only of non-tilde operators. Needless to say that observable operators are bosonic and Hermitian. We can confirm that the expectation value of an observable operator $A$ is real as

$$
\begin{aligned}
\langle A\rangle_{t}^{*} & =(\langle 1|A| 0(t)\rangle)^{\sim}=\sim\langle 1|\tilde{A}| 0(t)\rangle^{\sim}=\langle 1|\tilde{A}| 0(t)\rangle \\
& =\left\langle 1\left|A^{\dagger}\right| 0(t)\right\rangle=\langle 1|A| 0(t)\rangle=\langle A\rangle_{t} .
\end{aligned}
$$

Here we remark that the relation between NETFD and the formalism with quantum Liouville equation (1) is given through the "principle of correspondence"

$$
|0(t)\rangle \longleftrightarrow \rho(t), \quad\left\langle 1\left|\longleftrightarrow \operatorname{tr}, \quad A_{1} \tilde{A}_{2}^{\dagger}\right| 0(t)\right\rangle \longleftrightarrow A_{1} \rho(t) A_{2}
$$

The time evolution of a system is described by the Schrödinger equation (7) within NETFD with the initial condition $|0(t=0)\rangle=|0\rangle$. (The equation (7) corresponds to (1).) The infinitesimal time-evolution generator $\hat{H}$, called hat-Hamiltonian, satisfies the condition,

$$
(\mathrm{i} \hat{H})^{\sim}=\mathrm{i} \hat{H} \text {. }
$$

${ }^{4}$ We follow [11,12] for the double tilde conjugation rule (10) and TSC (17), since they are the most general definitions. Note that they are different from those in the original version [5-7]. See [12] for the derivation of (10) and (17). 
(The equation (21) corresponds to (2).) Note that the operators satisfying the condition (21) are called tildian operators that are not necessarily Hermitian. The tildian hat-Hamiltonian has zero eigenvalue for the thermal bra-vacuum:

$$
\langle 1| \hat{H}=0,
$$

which ensures the conservation of probability (16). (The equation (22) corresponds to (3).) The expectation value of an operator $A$ can be written as

$$
\langle A\rangle_{t}=\langle 1|A| 0(t)\rangle=\left\langle 1\left|\mathrm{e}^{\mathrm{i} \hat{H} t} A \mathrm{e}^{-\mathrm{i} \hat{H} t}\right| 0\right\rangle=\langle 1|A(t)| 0\rangle,
$$

where we have used (22) and the formal solution

$$
|0(t)\rangle=\mathrm{e}^{-\mathrm{i} \hat{H} t}|0\rangle
$$

of (7) for the second equality. (The equation (23) corresponds to (4).) The new operator

$$
A(t):=\mathrm{e}^{\mathrm{i} \hat{H} t} A \mathrm{e}^{-\mathrm{i} \hat{H} t}
$$

is the Heisenberg operator satisfying the Heisenberg equation,

$$
\frac{\mathrm{d}}{\mathrm{d} t} A(t)=\mathrm{i}[\hat{H}(t), A(t)]
$$

with $\hat{H}(t)=\mathrm{e}^{\mathrm{i} \hat{H} t} \hat{H} \mathrm{e}^{-\mathrm{i} \hat{H} t}=\hat{H}$. (The equations (25) and (26) correspond, respectively, to (5) and (6).)

\subsection{Semi-free hat-Hamiltonian}

Let $a_{i}, a_{i}^{\dagger}, \tilde{a}_{i}$ and $\tilde{a}_{i}^{\dagger}$ be operators satisfying the canonical (anti-)commutation relations

$$
\left[a_{i}, a_{j}^{\dagger}\right]_{-\sigma}=\delta_{i j}, \quad\left[\tilde{a}_{i}, \tilde{a}_{j}^{\dagger}\right]_{-\sigma}=\delta_{i j},
$$

where $\sigma=1(-1)$ for bosonic (fermionic) systems. Here, the subscripts $i$ and $j$ signify momentum, spin and/or other degrees of freedom. In what follows in this paper, we consider operators belonging to a specific index, say, $i$, and, therefore, suppress it for simplicity unless they are necessary.

When a physical particle is subject to dissipation, it has a finite life-time. The field which corresponds to a particle with finite life-time is called a semi-free field. The hat-Hamiltonian for a semi-free field is given by $[5-7,12]$

$$
\begin{aligned}
\hat{H} & =\hat{H}_{S}+\mathrm{i} \hat{\Pi}, \\
\hat{H}_{S} & =\omega\left(a^{\dagger} a-\tilde{a}^{\dagger} \tilde{a}\right), \\
\hat{\Pi} & =-\kappa\left[(1+2 \sigma \bar{n})\left(a^{\dagger} a+\tilde{a}^{\dagger} \tilde{a}\right)-2 \tau(\sigma+\bar{n}) a \tilde{a}-2 \tau \sigma \bar{n} a^{\dagger} \tilde{a}^{\dagger}+2 \bar{n}\right],
\end{aligned}
$$

with time-independent real numbers $\omega$ and $\kappa(>0) .{ }^{5}$ Note that the hat-Hamiltonian of the semi-free field is bilinear in $a, a^{\dagger}, \tilde{a}$ and $\tilde{a}^{\dagger}$, and is invariant under the global phase transformation $a \rightarrow a \mathrm{e}^{\mathrm{i} \phi}$ with a real parameter $\phi$. Note also that the hat-Hamiltonian satisfies the properties (21) and (22).

The equation of motion for the one-particle distribution function

$$
n(t):=\left\langle 1\left|a^{\dagger} a\right| 0(t)\right\rangle
$$

is given by

$$
\frac{\mathrm{d}}{\mathrm{d} t} n(t)=-2 \kappa[n(t)-\bar{n}]
$$

In deriving (32), TSC (17) with $A=a$, i.e.,

$$
\tau\langle 1| \tilde{a}=\langle 1| a^{\dagger},
$$

is used. The equation (32) is solved, with the initial condition $n(0)=n$, to give

$$
n(t)=\bar{n}+(n-\bar{n}) \mathrm{e}^{-2 \kappa t} .
$$

We see that $n(t)$ relaxes to $\bar{n}(\geqslant 0)$ for $t \rightarrow \infty$. Note that the information of the final state is included in $\hat{H}$ through $\bar{n}$.

\footnotetext{
${ }^{5}$ When a system is under the effect of non-stationary environment, $\omega$ and $\kappa$ may depend on time (see [9]).
} 


\subsection{Annihilation operators at the initial time}

Let the initial ket-vacuum $|0\rangle=|0(t=0)\rangle$ be specified by TSC

$$
\tilde{a}|0\rangle=\tau f a^{\dagger}|0\rangle
$$

with a real number $f$. The relation between $f$ and the initial one-particle distribution function $n$ can be derived from two different expressions for the expectation $\langle 1|a \tilde{a}| 0\rangle$ :

$$
\langle 1|a \tilde{a}| 0\rangle=\tau f\left\langle 1\left|a a^{\dagger}\right| 0\right\rangle=\tau f[\sigma n+1]
$$

and

$$
\langle 1|a \tilde{a}| 0\rangle=\sigma\langle 1|\tilde{a} a| 0\rangle=\sigma \tau^{-1}\left\langle 1\left|a^{\dagger} a\right| 0\right\rangle=\tau n
$$

These expressions yield

$$
n=\frac{f}{1-\sigma f}, \quad\left(f=\frac{n}{1+\sigma n}\right) .
$$

If the system is in thermal equilibrium with temperature $T_{0}$ at the initial time $t=0$, then $n$ is given by

$$
n=\frac{1}{\mathrm{e}^{\omega / T_{0}}-\sigma}
$$

which implies $f=\mathrm{e}^{-\omega / T_{0}}$.

The annihilation operators $\gamma_{0}\left(=\gamma_{t=0}\right)$ and $\tilde{\gamma}_{0}\left(=\tilde{\gamma}_{t=0}\right)$ associated with the initial vacua $|0\rangle$ $(=|0(t=0)\rangle)$, and the creation operators $\gamma^{+}$and $\tilde{\gamma}^{+}$associated with the bra-vacuum $\langle 1|$, i.e.,

$$
\gamma_{0}|0\rangle=0, \quad \tilde{\gamma}_{0}|0\rangle=0, \quad\langle 1| \gamma^{+}=0, \quad\langle 1| \tilde{\gamma}^{+}=0
$$

are given through the Bogoliubov transformation

$$
\left(\begin{array}{c}
\gamma_{0} \\
\tilde{\gamma}^{\dagger}
\end{array}\right):=\left(\begin{array}{cc}
1+\sigma n & -\tau \sigma n \\
-\tau \sigma & 1
\end{array}\right)\left(\begin{array}{c}
a \\
\tilde{a}^{\dagger}
\end{array}\right)
$$

and its tilde conjugate. They satisfy the canonical (anti-)commutation relations

$$
\left[\gamma_{0}, \gamma^{+}\right]_{-\sigma}=1, \quad\left[\tilde{\gamma}_{0}, \tilde{\gamma}^{+}\right]_{-\sigma}=1
$$

As was indicated before, $\gamma^{+}$and $\tilde{\gamma}^{+}$do not have a subscript indicating time. This is a manifestation of the fact that $\langle 1|$ does not depend on time.

\section{Annihilation operators associated with the unstable vacuum}

Let $\left\{\gamma_{0}^{(i)}, \gamma^{(i)^{+}}\right\}_{i=1}^{M}$ be a set of annihilation and creation operators associated with the initial vacua $|0\rangle$ and $\langle 1|$ :

$$
\begin{aligned}
& \gamma_{0}^{(i)}|0\rangle=0, \quad\langle 1| \gamma^{(i)^{+}}=0, \\
& {\left[\gamma_{0}^{(i)}, \gamma^{(j)^{+}}\right]_{-\sigma}=\delta_{i j},} \\
& {\left[\gamma_{0}^{(i)}, \gamma_{0}^{(j)}\right]_{-\sigma}=0, \quad\left[\gamma^{(i)^{+}}, \gamma^{(j)^{+}}\right]_{-\sigma}=0 .}
\end{aligned}
$$

Equations (40) and (42) correspond to $M=2$ with

$$
\gamma_{0}^{(1)}=\gamma_{0}, \quad \gamma^{(1)^{+}}=\gamma^{+}, \quad \gamma_{0}^{(2)}=\tilde{\gamma}_{0}, \quad \gamma^{(2)^{+}}=\tilde{\gamma}^{+} .
$$

Here, we proceed with $M$ being an arbitrary positive integer. 
Let us start the discussion with the hat-Hamiltonian for a semi-free field:

$$
\hat{H}=g_{1}^{i j} \gamma_{0}^{(i)} \gamma_{0}^{(j)}+g_{2}^{i j} \gamma^{(i)^{+}} \gamma_{0}^{(j)}+g_{3}^{i j} \gamma^{(i)^{+}} \gamma^{(j)^{\circ}}+g_{0},
$$

written in the form of the normal-ordering with respect to the creation and annihilation operators. Here, $g_{k}^{i j}$ are time-independent c-numbers. We are adopting here the summation rule for repeated indices unless otherwise stated.

Our purpose is to find the set of annihilation operators $\gamma_{t}^{(i)}$ associated with the vacuum $|0(t)\rangle$ at time $t$ specified by

$$
\begin{aligned}
& \gamma_{t}^{(i)}|0(t)\rangle=0 \\
& {\left[\gamma_{t}^{(i)}, \gamma^{(j)^{+}}\right]_{-\sigma}=\delta_{i j}, \quad\left[\gamma_{t}^{(i)}, \gamma_{t}^{(j)}\right]_{-\sigma}=0 .}
\end{aligned}
$$

Here, the vacuum $|0(t)\rangle$ is the solution of the Schrödinger equation (7) with the hat-Hamiltonian (47).

Let us introduce "Heisenberg operators" associated with the operators $\gamma_{s}^{(i)}$ and $\gamma^{(i)^{+}}$by

$$
\gamma_{s}^{(i)}(t):=\mathrm{e}^{\mathrm{i} \hat{H} t} \gamma_{s}^{(i)} \mathrm{e}^{-\mathrm{i} \hat{H} t}, \quad \gamma^{(i)^{+}}(t):=\mathrm{e}^{\mathrm{i} \hat{H} t} \gamma^{(i)^{+}} \mathrm{e}^{-\mathrm{i} \hat{H} t}
$$

Note that "Heisenberg operator" $\gamma_{s}^{(i)}(t)$ has two time indices $s$ and $t$, since the corresponding "Schrödinger operator" $\gamma_{s}^{(i)}$ depends on time $s$ due to the time-dependence of the vacuum $|0(s)\rangle$ $(\operatorname{see}(48))$.

Using the first equations in (43) and (50), we have

$$
0=\mathrm{e}^{-\mathrm{i} \hat{H} t} \gamma_{0}^{(i)}|0\rangle=\mathrm{e}^{-\mathrm{i} \hat{H} t} \gamma_{0}^{(i)} \mathrm{e}^{\mathrm{i} \hat{H} t} \mathrm{e}^{-\mathrm{i} \hat{H} t}|0\rangle=\gamma_{0}^{(i)}(-t)|0(t)\rangle
$$

Thus, we notice that the $M$ operators $\gamma_{0}^{(i)}(-t)(i=1, \cdots, M)$ should be related to the annihilation operators $\gamma_{t}^{(i)}(i=1, \cdots, M)$ satisfying (48). Since an arbitrary linear combination of $\gamma_{0}^{(i)}(-t)$ also satisfies (48), let us find $\gamma_{t}^{(i)}$ which satisfies both (48) and (49) in the form

$$
\gamma_{t}^{(i)}=C^{i j}(t) \gamma_{0}^{(j)}(-t)
$$

where $C^{i j}(t)(i, j=1, \cdots, M)$ are time-dependent c-number functions. It is easy to check that $\gamma_{t}^{(i)}$ of the form (52) satisfies the second equation in (49) as follows.

$$
\left[\gamma_{t}^{(i)}, \gamma_{t}^{(j)}\right]_{-\sigma}=C^{i k}(t) C^{j l}(t)\left[\gamma_{0}^{(k)}(-t), \gamma_{0}^{(l)}(-t)\right]_{-\sigma}=C^{i k}(t) C^{j l}(t) \mathrm{e}^{-\mathrm{i} \hat{H} t}\left[\gamma_{0}^{(k)}, \gamma_{0}^{(l)}\right]_{-\sigma} \mathrm{e}^{\mathrm{i} \hat{H} t}=0
$$

Now, the second equation of (43) can be rewritten in terms of the second operator in (50) as

$$
0=\langle 1| \gamma^{(i)^{+}} \mathrm{e}^{-\mathrm{i} \hat{H} t}=\langle 1| \mathrm{e}^{\mathrm{i} \hat{H} t} \gamma^{(i)^{+}} \mathrm{e}^{-\mathrm{i} \hat{H} t}=\langle 1| \gamma^{(i)^{+}}(t) .
$$

At the second equality, (22) has been used. The creation operator $\gamma^{(i)^{+}}(t)$ is the solution of the Heisenberg equation

$$
\frac{\mathrm{d}}{\mathrm{d} t} \gamma^{(i)^{+}}(t)=\mathrm{i}\left[\hat{H}(t), \gamma^{(i)^{+}}(t)\right]
$$

In virtue of the bilinear form $(47)$ of $\hat{H}, \gamma^{(i)^{+}}(t)$ can be given by a linear combination of $\gamma_{0}^{(j)}$ and $\gamma^{(j)^{+}}(j=1, \cdots, M)$. Equation (54) entails that only $\gamma^{(j)^{+}}$can be a member of the linear combination, that is,

$$
\gamma^{(i)^{+}}(t)=\gamma^{(j)^{+}} F^{j i}(t)
$$

where $F^{j i}(t)(i, j=1, \cdots, M)$ are time-dependent c-number functions. Let $F(t)$ denote $M \times M$ matrix whose $(i, j)$-element is given by $F^{i j}(t)$, and $F(t)^{-1}$ is the inverse matrix of $F(t)$. Then, we have

$$
\gamma^{(i)^{+}}=\gamma^{(j)^{+}}(t)\left(F(t)^{-1}\right)^{j i} .
$$


For the first equation in (49) to be satisfied, it is required that

$$
\begin{aligned}
\delta_{i j} & =\left[\gamma_{t}^{(i)}, \gamma^{(j)^{+}}\right]_{-\sigma} \\
& =C^{i k}(t)\left[\gamma_{0}^{(k)}(-t), \gamma^{(l)^{+}}(-t)\right]_{-\sigma}\left(F(-t)^{-1}\right)^{l j} \\
& =C^{i k}(t) \mathrm{e}^{-\mathrm{i} \hat{H} t}\left[\gamma_{0}^{(k)}, \gamma^{(l)^{+}}\right]_{-\sigma} \mathrm{e}^{\mathrm{i} \hat{H} t}\left(F(-t)^{-1}\right)^{l j} \\
& =C^{i k}(t) \delta_{k l}\left(F(-t)^{-1}\right)^{l j} \\
& =C^{i k}(t)\left(F(-t)^{-1}\right)^{k j}
\end{aligned}
$$

which leads us to

$$
C^{i j}(t)=F^{i j}(-t)
$$

In deriving (58), we have used (44), (52) and (57).

Substituting (59) into (52), we finally arrive at the desired annihilation operators in the form

$$
\gamma_{t}^{(i)}=F^{i j}(-t) \gamma_{0}^{(j)}(-t)
$$

Here, $F(-t)^{i j}$ is given through $(56)$, and $\gamma_{0}^{(j)}(-t)$ by solving the "Heisenberg equation" for $\gamma_{0}^{(j)}(t)$ :

$$
\frac{\mathrm{d}}{\mathrm{d} t} \gamma_{0}^{(j)}(t)=\mathrm{i}\left[\hat{H}(t), \gamma_{0}^{(j)}(t)\right]
$$

\section{Example: Semi-free fields}

Let us consider the case when $\hat{H}$ is the semi-free hat-Hamiltonian given by (28) with (29) and (30). The number of the sets of annihilation and creation operators $M$ is 2 and the annihilation and creation operators are given by (46). By using inverse transformation of (41), $\hat{H}_{\mathrm{S}}$ in $(29)$ and $\hat{\Pi}$ in $(30)$ can be written in terms of $\gamma_{0}, \gamma^{+}, \tilde{\gamma}_{0}$ and $\tilde{\gamma}^{+}$as

$$
\begin{aligned}
\hat{H}_{\mathrm{S}} & =\omega\left(\gamma^{+} \gamma_{0}-\tilde{\gamma}^{+} \tilde{\gamma}_{0}\right), \\
\hat{\Pi} & =-\kappa\left[\gamma^{+} \gamma_{0}+\tilde{\gamma}^{+} \tilde{\gamma}_{0}+2 \tau \sigma(n-\bar{n}) \gamma^{+} \tilde{\gamma}^{+}\right] .
\end{aligned}
$$

The "Heisenberg equations" for $\gamma_{0}(t)$ and $\gamma^{+}(t)$ are given by

$$
\begin{aligned}
\frac{\mathrm{d}}{\mathrm{d} t} \gamma_{0}(t) & =\mathrm{i}\left[\hat{H}(t), \gamma_{0}(t)\right]=(-i \omega-\kappa) \gamma_{0}(t)-2 \tau \sigma \kappa(n-\bar{n}) \tilde{\gamma}^{+}(t), \\
\frac{\mathrm{d}}{\mathrm{d} t} \gamma^{+}(t) & =\mathrm{i}\left[\hat{H}(t), \gamma^{+}(t)\right]=(\mathrm{i} \omega+\kappa) \gamma^{+}(t),
\end{aligned}
$$

and are solved with the initial condition $\gamma_{0}(0)=\gamma_{0}$ and $\gamma^{+}(0)=\gamma^{+}$as

$$
\begin{aligned}
\gamma_{0}(t) & =\mathrm{e}^{(-\mathrm{i} \omega-\kappa) t}\left[\gamma_{0}+\tau \sigma(n-\bar{n})\left(1-\mathrm{e}^{2 \kappa t}\right) \tilde{\gamma}^{\dagger}\right], \\
\gamma^{+}(t) & =\mathrm{e}^{(\mathrm{i} \omega+\kappa) t} \gamma^{\dagger} .
\end{aligned}
$$

Equation (67) implies

$$
\left(\begin{array}{cc}
\gamma^{+}(t) & \tilde{\gamma}^{+}(t)
\end{array}\right)=\left(\begin{array}{cc}
\gamma^{+} & \tilde{\gamma}^{+}
\end{array}\right) F(t)
$$

with

$$
F(t)=\left(\begin{array}{cc}
\mathrm{e}^{(\mathrm{i} \omega+\kappa) t} & 0 \\
0 & \mathrm{e}^{(-\mathrm{i} \omega+\kappa) t}
\end{array}\right)
$$

From (60), (66) and (69), we obtain $\gamma_{t}$ and $\tilde{\gamma}_{t}$ as

$$
\left(\begin{array}{c}
\gamma_{t} \\
\tilde{\gamma}_{t}
\end{array}\right)=F(-t)\left(\begin{array}{c}
\gamma_{0}(-t) \\
\tilde{\gamma}_{0}(-t)
\end{array}\right)=\left(\begin{array}{c}
\gamma_{0} \\
\tilde{\gamma}_{0}
\end{array}\right)+(n-\bar{n})\left(1-\mathrm{e}^{-2 \kappa t}\right)\left(\begin{array}{c}
\tau \sigma \tilde{\gamma}^{q} \\
\tau \gamma^{q}
\end{array}\right) .
$$


By using (34), (41) and (70), $\gamma_{t}$ and $\gamma^{+}$can be expressed in terms of $a, a^{\dagger}, \tilde{a}$ and $\tilde{a}^{\dagger}$ as

$$
\begin{aligned}
\left(\begin{array}{c}
\gamma_{t} \\
\tilde{\gamma}^{+}
\end{array}\right) & =\left(\begin{array}{cc}
1 & \tau \sigma(n-\bar{n})\left(1-\mathrm{e}^{-2 \kappa t}\right) \\
0 & 1
\end{array}\right)\left(\begin{array}{c}
\gamma_{0} \\
\tilde{\gamma}^{+}
\end{array}\right) \\
& =\left(\begin{array}{cc}
1+\sigma n(t) & -\tau \sigma n(t) \\
-\tau \sigma & 1
\end{array}\right)\left(\begin{array}{c}
a \\
\tilde{a}^{\dagger}
\end{array}\right)
\end{aligned}
$$

(and its tilde conjugate), which is a time-dependent Bogoliubov transformation. The time-dependent Bogoliubov transformation was first obtained in a slightly different form in [5-7]. We have derived the transformation following the new route given above.

Using (34), (62), (63) and (70), we can rewrite $\hat{H}_{\mathrm{S}}$ and $\hat{\Pi}$ in terms of $\gamma_{t}, \gamma^{+}, \tilde{\gamma}_{t}$ and $\tilde{\gamma}^{+}$as

$$
\begin{aligned}
\hat{H}_{\mathrm{S}} & =\omega\left(\gamma^{+} \gamma_{t}-\tilde{\gamma}^{+} \tilde{\gamma}_{t}\right), \\
\hat{\Pi} & =-\kappa\left\{\gamma^{+} \gamma_{t}+\tilde{\gamma}^{+} \tilde{\gamma}_{t}+2 \tau \sigma[n(t)-\bar{n}] \gamma^{+} \tilde{\gamma}^{+}\right\} .
\end{aligned}
$$

By substituting (28) with (72) and (73) into the Schrödinger equation (7), we have

$$
\frac{\mathrm{d}}{\mathrm{d} t}|0(t)\rangle=-2 \tau \sigma \kappa[n(t)-\bar{n}] \gamma^{+} \tilde{\gamma}^{+}|0(t)\rangle=\tau \sigma \frac{\mathrm{d} n(t)}{\mathrm{d} t} \gamma^{+} \tilde{\gamma}^{+}|0(t)\rangle,
$$

where we used (32). The solution is given by

$$
|0(t)\rangle=\exp \left\{\tau \sigma[n(t)-\bar{n}] \gamma^{+} \tilde{\gamma}^{+}\right\}|0\rangle .
$$

This expression, which was first obtained in [9], implies that the time evolution of the unstable vacuum $|0(t)\rangle$ can be interpreted as the condensation of $\gamma^{+} \tilde{\gamma}^{q}$-pairs into the vacuum.

\section{Discussion}

In this paper, we presented an alternative route of deriving the set of annihilation operators $\left\{\gamma_{t}^{(i)}\right\}_{i=1}^{M}$ at time $t$ satisfying (48), $\gamma_{t}^{(i)}|0(t)\rangle=0$, starting with the set of annihilation operators $\left\{\gamma_{0}^{(i)}\right\}_{i=1}^{M}$ at the initial time $t=0$ satisfying the first equation in (43), $\gamma_{0}^{(i)}|0\rangle=0$, under the condition that the vacuums $|0(t)\rangle$ and $|0\rangle$ are related with each other by $(24),|0(t)\rangle=\mathrm{e}^{-\mathrm{i} \hat{H} t}|0\rangle$. This setup of the problem reminds us of the path in QFT to derive the annihilation operators parameterized by a real number $\theta$ through a unitary operator $U(\theta)$. Let $|0\rangle$ be a vacuum that is annihilated by the annihilation operator $a$, i.e., $a|0\rangle=0$. The procedure

$$
0=U(\theta) a|0\rangle=U(\theta) a U^{-1}(\theta) U(\theta)|0\rangle=a(\theta)|0(\theta)\rangle
$$

gives the new annihilation operator

$$
a(\theta):=U(\theta) a U^{-1}(\theta)
$$

that annihilates the new vacuum

$$
|0(\theta)\rangle=U(\theta)|0\rangle
$$

Note that (51) is quite similar to $(76)$ when $U(\theta)$ is replaced by $\mathrm{e}^{-\mathrm{i} \hat{H} t}, a$ by $\gamma_{0}^{(i)}$ and $a(\theta)$ by $\gamma_{0}^{(i)}(-t)$. This formal similarity is due to the fact that both QFT and NETFD form canonical operator formalisms. Note also that the operator $\gamma_{0}^{(i)}(-t)$ appears in (51) instead of the annihilation operator $\gamma_{t}^{(i)}$. This is a reflection of the fact that the bra-vacuum $\langle 1|$ in NETFD is independent of time. It follows that the creation operators $\gamma^{(i)^{+}}$are independent of time and that, in order to satisfy the canonical (anti-) commutation relation $\left[\gamma_{t}^{(i)}, \gamma^{(j)^{+}}\right]_{-\sigma}=\delta_{i j}$, i. e., the first equation of (49), $\gamma_{t}^{(i)}$ should not be $\gamma_{0}^{(i)}(-t)$ itself but a linear combination of $\gamma_{0}^{(j)}(-t)$ given by $(60)$. 
It was revealed in this paper that a special care is required when the parameter $\theta$ in $U(\theta)$ labeling vacua turns out to be time $t$ appearing in the time evolution operator $\mathrm{e}^{-\mathrm{i} \hat{H} t}$. One needs to deal with the annihilation operator in the "Heisenberg representation" containing two times, $s$ and $t$, defined by the first equation in $(50), \gamma_{s}^{(i)}(t)=\mathrm{e}^{\mathrm{i} \hat{H} t} \gamma_{s}^{(i)} \mathrm{e}^{-\mathrm{i} \hat{H} t}$. Note that, for $s=t$, it reduces to the annihilation operator $\gamma^{(i)}(t)=\mathrm{e}^{\mathrm{i} \hat{H} t} \gamma_{t}^{(i)} \mathrm{e}^{-\mathrm{i} \hat{H} t}$ which annihilates the initial vacuum $|0\rangle$, i.e., $\gamma^{(i)}(t)|0\rangle=0$. As is given in appendix A, the annihilation operators $\gamma_{t}^{(i)}$ associated with the unstable vacuum $|0(t)\rangle$ were conventionally introduced in relation to $\gamma^{(i)}(t)=\gamma_{t}^{(i)}(t)$, whereas $\gamma_{t}^{(i)}$ are given in relation to $\gamma_{0}^{(i)}(-t)$ as $(60)$ in the new route given in this paper.

It should be noted here that, in QFT, the two representation spaces created on vacua $|0\rangle$ and $|0(\theta)\rangle$ are unitarily inequivalent to each other, and that the operation of the unitary operator $U(\theta)$ in (78) which relates the two vacua should be considered as a formal expression. In spite of the fact that the expansion of $|0(\theta)\rangle$ by means of the states in the unitarily inequivalent representation space created on $|0\rangle$ is inappropriate, one can interpret a physical relation between the vacua $|0\rangle$ and $|0(\theta)\rangle$, i.e., since the operation of $U(\theta)$ on $|0\rangle$ can be given in terms of the creation operators $a_{i}^{\dagger}$ and/or their pairs $a_{i}^{\dagger} a_{j}^{\dagger}$ (the subscripts $i, j$ signify degrees of freedom such as momentum, spin, etc.), the expression (78) tells us that $|0(\theta)\rangle$ is created by condensing infinitely many $a_{i}^{\dagger}$-particles and/or $a_{i}^{\dagger} a_{j}^{\dagger}$-pairs into $|0\rangle$ (see, e.g., [13]). The physical picture of condensation of particles and/or pairs in vacuum holds in NETFD as well. As is given in section 4, for systems of semi-free fields, the operation of $\mathrm{e}^{-\mathrm{i} \hat{H} t}$ on $|0\rangle$ reduces to the expression (75) which indicates the condensation of $\gamma^{+} \tilde{\gamma}^{+}$-pairs into $|0\rangle$ providing us with $|0(t)\rangle$. Note that the time-evolution operator $\mathrm{e}^{-\mathrm{i} \hat{H} t}$ which relates $|0(t)\rangle$ to $|0\rangle$ in $(24)$ is not necessarily unitary since the hat-Hamiltonian $\hat{H}$ is not always Hermitian due to the dissipation part îิ.

The derivation of annihilation operators of the unstable vacuum via the present route can be applied to those systems containing dynamical symmetry breaking in addition to the dissipative evolution $[9,14,15]$. One of the examples is the system subject to both squeezing and dissipation. The analysis of annihilation operators in the system will be reported elsewhere in the near future.

\section{Appendix}

\section{A. Conventional derivation of annihilation operators associated with unsta- ble vacuum}

In this section, we review the conventional derivation of annihilation operators associated with an unstable vacuum $|0(t)\rangle[5-7,9]$. The system of semi-free field, i.e., unstable physical particles, is specified by $\hat{H}$ given in (28) with (29) and (30). Let us introduce the Heisenberg operators associated with $a, a^{\dagger}, \tilde{a}$ and $\tilde{a}^{\dagger}$ by

$$
\begin{array}{ll}
a(t):=\mathrm{e}^{\mathrm{i} \hat{H} t} a \mathrm{e}^{-\mathrm{i} \hat{H} t}, & a^{\#}(t):=\mathrm{e}^{\mathrm{i} \hat{H} t} a^{\dagger} \mathrm{e}^{-\mathrm{i} \hat{H} t}, \\
\tilde{a}(t):=\mathrm{e}^{\mathrm{i} \hat{H} t} \tilde{a} \mathrm{e}^{-\mathrm{i} \hat{H} t}, & \tilde{a}^{\dagger}(t):=\mathrm{e}^{\mathrm{i} \hat{H} t} \tilde{a}^{\dagger} \mathrm{e}^{-\mathrm{i} \hat{H} t} .
\end{array}
$$

The notations $a^{\#}(t)$ and $\tilde{a}^{\#}(t)$ were introduced since they are not always equal to $(a(t))^{\dagger}$ and $(\tilde{a}(t))^{\dagger}$ due to the fact that $\hat{H}$ is not necessarily Hermitian. Solving the Heisenberg equations for $a(t), a^{\#}(t), \tilde{a}(t)$ and $\tilde{a}^{\#}(t)$, we have

$$
\left(\begin{array}{c}
a(t) \\
\tilde{a}^{\#}(t)
\end{array}\right)=B^{-1}\left(\begin{array}{cc}
\mathrm{e}^{(-\mathrm{i} \omega-\kappa) t} & 0 \\
0 & \mathrm{e}^{(-\mathrm{i} \omega+\kappa) t}
\end{array}\right) B\left(\begin{array}{c}
a \\
\tilde{a}^{\dagger}
\end{array}\right),
$$

and its tilde conjugate, where

$$
B:=\left(\begin{array}{cc}
1+\bar{n} \sigma & -\tau \bar{n} \sigma \\
-\tau \sigma & 1
\end{array}\right)
$$


Substituting $a, \tilde{a}^{\dagger}$ and their tilde conjugates, given by the inverse transformation of (81), into TSCs (33) and (35), we obtain

$$
\left\langle 1\left|\left[-\tau \sigma a(t)+\tilde{a}^{\#}(t)\right]=0, \quad\left[(1+\sigma n(t)) a(t)-\tau \sigma n(t) \tilde{a}^{\#}(t)\right]\right| 0\right\rangle=0,
$$

where we have used (34). Hence, we find the annihilation operators $\gamma(t)$ and $\tilde{\gamma}(t)$ and the creation operators $\gamma^{+}(t)$ and $\tilde{\gamma}^{+}(t)$ for unstable physical particles satisfying

$$
\gamma(t)|0\rangle=0, \quad \tilde{\gamma}(t)|0\rangle=0, \quad\langle 1| \gamma^{+}(t)=0, \quad\langle 1| \tilde{\gamma}^{\ddagger}(t)=0,
$$

with the canonical (anti-)commutation relations

$$
\left[\gamma(t), \gamma^{+}(t)\right]_{-\sigma}=1, \quad\left[\tilde{\gamma}(t), \tilde{\gamma}^{+}(t)\right]_{-\sigma}=1,
$$

through the time-dependent Bogoliubov transformation

$$
\left(\begin{array}{c}
\gamma(t) \\
\tilde{\gamma}^{+}(t)
\end{array}\right):=\left(\begin{array}{cc}
1+\sigma n(t) & -\tau \sigma n(t) \\
-\tau \sigma & 1
\end{array}\right)\left(\begin{array}{c}
a(t) \\
\tilde{a}^{\dagger}(t)
\end{array}\right)
$$

and its tilde conjugate.

The annihilation operators $\gamma_{t}$ and $\tilde{\gamma}_{t}$ and the creation operators $\gamma^{+}$and $\tilde{\gamma}^{+}$associated with the vacua $|0(t)\rangle$ and $\langle 1|$ specified by

$$
\gamma_{t}|0(t)\rangle=0, \quad \tilde{\gamma}_{t}|0(t)\rangle=0, \quad\langle 1| \gamma^{\text {क }}=0, \quad\langle 1| \tilde{\gamma}^{\text {क }}=0,
$$

are derived from (84) with the relations

$$
\begin{aligned}
& \gamma(t)=\mathrm{e}^{\mathrm{i} \hat{H} t} \gamma_{t} \mathrm{e}^{-\mathrm{i} \hat{H} t}, \quad \tilde{\gamma}(t)=\mathrm{e}^{\mathrm{i} \hat{H} t} \tilde{\gamma}_{t} \mathrm{e}^{-\mathrm{i} \hat{H} t}, \\
& \gamma^{+}(t)=\mathrm{e}^{\mathrm{i} \hat{H} t} \gamma^{\uparrow} \mathrm{e}^{-\mathrm{i} \hat{H} t}, \quad \tilde{\gamma}^{q}(t)=\mathrm{e}^{\mathrm{i} \hat{H} t} \tilde{\gamma}^{+} \mathrm{e}^{-\mathrm{i} \hat{H} t} .
\end{aligned}
$$

They satisfy the Bogoliubov transformation (71) and the canonical (anti-)commutation relation

$$
\left[\gamma_{t}, \gamma^{+}\right]_{-\sigma}=1, \quad\left[\tilde{\gamma}_{t}, \tilde{\gamma}^{+}\right]_{-\sigma}=1 .
$$

\section{References}

1. Prigogine I. Non-equilibrium Statistical Mechanics. Interscience Publisher, 1962.

2. Crawford J.A., Nuovo Cim., 1958, 10, 698-713.

3. Haake F., Springer Tracts Mod. Phys., 1973, 66, 98-168.

4. Agarwal G.S., Wolf E., Phys. Rev. D, 1970, 2, 2161; 2187; 2206.

5. Arimitsu T., Umezawa H., Prog. Theor. Phys., 1985, 74, 429-432.

6. Arimitsu T., Umezawa H., Prog. Theor. Phys., 1987, 77, 32-52.

7. Arimitsu T., H. Umezawa., Prog. Theor. Phys., 1987, 77, 53-67.

8. Umezawa H., Matsumoto H., Tachiki M. Thermo Field Dynamics and Condensed States. NorthHolland Publishing Company, 1982.

9. Arimitsu T., Sudo Y., Umezawa H., Physica A, 1987, 146, 433-451.

10. Arimitsu T., Condens. Matter Phys., 1994, 4, 26-88.

11. Ojima I., Ann. Phys. (N. Y.), 1981, 137, 1-32.

12. Kobryn A.E., Hayashi T., Arimitsu T., Ann. Phys., 2003, 308, 395-446.

13. Umezawa H. Advanced Field Theory Micro, Macro, and Thermal Physics. American Institute of Physics, New York, 1993.

14. Arimitsu T., Guida M., Umezawa H., Physica A, 1988, 148, 1-26.

15. Tominaga T., Ban M., Arimitsu T., Pradko J. Umezawa H., Physica A, 1988, 149, 26-481. 


\title{
Оператори анігіляції, пов'язані з нестабільним вакуумом в нерівноважній термопольовій динаміці
}

\author{
К.Йошіда, Т.Аріміцу \\ Вища школа фундаментальних і прикладних наук, університет м. Цукуба, Ібаракі, Японія \\ Отримано 14 липня 2008 р.
}

Далеко від стану термічної рівноваги вакуум є нестабільним і змінюється з часом. В результаті, оператори анігіляції, пов'язані з нестабільним вакуумом, еволюціонують з часом. Ця дисипативна часова еволюція квантових систем може бути систематично досліджена в рамках формалізму канонічного оператора, який має назву нерівноважна термо-польова динаміка. В рамках цього формалізму запропоновано альтернативний шлях отримання залежних від часу операторів анігіляції. Як приклад, отримано залежні від часу оператори анігіляції для систем бозонних і ферміонних напіввільних полів.

Ключові слова: нерівноважна термопольова динаміка, оператор анігіляції, нестабільний вакуум, дисипативні квантові системи, часовозалежне перетворення Боголюбова

PACS: $11.10 . W x, 05.30 .-d$ 
